\title{
Affective Disorders and Antidepressant Drugs
}

\author{
Marc Fakhoury \\ Department of Neuroscience, Faculty of Medicine, University of Montreal, Montreal, Canada \\ Email: marc.fakhoury@umontreal.ca
}

Received 4 June 2014; revised 10 July 2014; accepted 18 August 2014

Copyright (C) 2014 by author and OALib.

This work is licensed under the Creative Commons Attribution International License (CC BY). http://creativecommons.org/licenses/by/4.0/

(c) (†) Open Access

\begin{abstract}
Affective disorders are a group of psychiatric diseases that can affect an individual at any given age. Also called mood disorders, they can be distinguished into two different types: major depressive disorder, also called major depression, and bipolar disorder, which is known as manic depression. People affected by major depression most often have a low mood, and are consistently in a state of unhappiness. Although it was shown that genetics play a role in the predisposition of depression, this disease most often occurs in response to a variety of external factors such as a stressful life event, the loss of a loved one, and following drug or substance abuse. A variety of antidepressant drugs, such as the monoamine oxidase inhibitors (MAOIs), the tricyclic antidepressants (TCAs), and the second-generation antidepressants are able to provide significant relief for people suffering from affective disorders like depression. However, several of these pharmaceutical agents can cause serious side effects to the patients. Therefore, there is a need to identify novel antidepressant therapies that are more efficient and that present minimal side effects. A better understanding of the neurobiology of depression will definitively help scientists develop new therapeutic ideas. This paper will first discuss the clinical profile of depression and explain the physiological mechanisms and the neurochemistry involved in this disease. It will then give you an overview of the effectiveness of the most common antidepressants used, with a description of their mode of action and most notable side effects.
\end{abstract}

\section{Keywords}

Antidepressant Drugs, Bipolar Depression, Depression, Neurobiology

Subject Areas: Drugs \& Devices, Neurology, Psychiatry \& Psychology

\section{Introduction}

Antidepressants are medications that are very useful in treating affective disorders such as depression, which is characterized by extreme and inappropriate exaggerations in mood, and an affective state of sadness. Antide- 
pressants are primarily prescribed because they have the ability to significantly enhance the mood of depressed patients relatively quickly. Typical signs and symptoms of depression include feeling of sadness, sleep disturbances (insomnia or hypersomnia), reduced appetite, and increased suicidality. Physical symptoms include localized pain, difficult breathing and severe digesting disturbances. Clinical depression can be found worldwide, and affects individuals of all ages and cultural origins. However, women tend to be more affected than men in general, which is often the result of experiencing a stressful event such as parental death, loss of a loved one, miscarriage or a divorce [1]. There are several factors that predispose someone getting affected by a mood disorder, and they are mainly genetic, psychosocial, and biological. Depression can also be the result of a stressful event, and can be precipitated by pharmacological agents or drug abuse [2]. Treatment of depressive disorders includes psychotherapy as well as the use of antidepressant drugs. Most of these drugs act by increasing the availability of specific brain chemicals called neurotransmitters, including dopamine and serotonin. It is believed that these brain chemicals can help improve emotions in depressed patients. The antidepressants commonly used to treat major depressive disorder are the monoamine oxidase inhibitors, the tricyclic antidepressants and the second-generation antidepressants. Most of these antidepressants work equally well to relieve depression and results can be seen after just couple of weeks of treatment. Although these pharmaceutical agents are useful in enhancing the symptoms of depressed patients, not every individual responds in the same manner to pharmacotherapy and some of these antidepressant drugs may present few or many side effects. This paper will give you an overview of the biological mechanisms implicated in affective disorders, and will discuss the effectiveness of antidepressant drugs and other pharmaceutical compounds in treating this disease.

\section{Clinical Profile of Depression: Demography, Signs and Subtypes}

Mood disorders are very common among individuals. For instance, the prevalence of depression in the United States can go up to $25 \%$ in women, and $12 \%$ in men [3]. Although this disease could occur at any age, the median age is about 40 years old. In contrast to depression, bipolar disorder is characterized by episodes of elevated mood known as mania, usually alternating with episodes of depression. This disorder affects men and women equally, the average age of onset being between 27 and 33 years old. Patients affected by depression often report signs such as loss of appetite, insomnia, loss of motivation and general fatigue. Low self-esteem and thoughts of suicide are also very common among depressed people. According to the Diagnostic and Statistical Manual of Mental Disorders [4], at least 5 of these symptoms must be present in order to diagnose someone from major depression: depressed mood most of the day, decreased interest or pleasure, change in weight and appetite, change in sleep, change in activity, loss of energy, feeling of worthlessness, diminished ability to concentrate and thoughts of death or suicide.

Recent research have tried to identify the subtypes of depression in order to better predict the effectiveness of a given antidepressant drug [5]. There exist six different types of depression: major depression with melancholia or endogenous features, major depression with melancholia (most common), major depression with psychotic features, bipolar depression, dysthymic disorder, and treatment-resistant depression. The most common form of depression is when it's experienced in conjunction with melancholia. Patients in this group of disease often exhibit signs such as anhedonia, emotional withdrawal and insomnia, but can be easily treated with tricyclic antidepressants. In contrast, antidepressant drugs are less effective with patients affected by major depression with psychotic features, unless they are administered concomitantly with an antipsychotic drug. People with bipolar disorder often need antidepressant drugs to alleviate the symptoms of depression, as well as a mood stabilizer such as lithium carbonate to control the mania. Dysthymic disorder, also referred to as premorbid neurotic depressive disorder, is a mood disorder consisting of the same cognitive and physical problems as seen in depression, with less severe but longer-lasting symptoms [6]. It is most effectively treated with tricyclic antidepressants after the patients have recovered from episodes of major depression [7]. Treatment-resistant depression is a term used in clinical psychiatry to describe cases of major depressive disorder that do not respond adequately appropriately to at least two antidepressants [8]. People in this category are often treated with a combination of two different types of antidepressants, or with the addition of a non-antidepressant medication that has the ability to enhance the effectiveness of the antidepressant.

\section{Physiological Mechanism of Depression}

1) Abnormal cortisol secretion in depressed patients. 
Cortisol is a steroid hormone produced by the zona fasciculata of the adrenal cortex. It is released in response to stress and a low level of blood glucocorticoid [9]. Altered patterns of serum cortisol levels have been observed in connection with abnormal adrenocorticotropic hormone (ACTH) levels, clinical depression and psychological stress. Approximately 50\% of the patients show an elevation of adrenal cortisol in response to a higher release of ACTH from the pituitary [10]. ACTH is an important component of the hypothalamic-pituitary-adrenal axis and is often produced in response to biological stress, and is regulated by the secretion of its precursor corticotropin-releasing hormone from the hypothalamus. It's also well believed that the abnormal cortisol levels found in depressed individuals are the result of a defect in the brain at or above the level of the hypothalamus [11]. In order to evaluate the function of the hypothalamic-pituitary-adrenal, scientists are using the dexamethasone suppression test, which assesses adrenal gland function by measuring how cortisol levels change in response to an injection of dexamethasone [12] [13]. In the depressed patients who were treated with dexamethasone, the plasma level of cortisol and beta-endorphin was found to be significantly reduced.

2) Biogenic amine hypothesis.

In the early 1950s, scientists tested several drugs that altered mood and behavior in order to evaluate their pharmacological mechanism of action. Reserpine, which reduces sympathetic constriction of blood vessels, was found to induce symptoms that are very similar to those found in depressed individuals. Because the reserpineinduced model of depression depletes stores of several monoamines such as dopamine, norepinephrine and serotonin [14] [15], the development of drugs that restore the normal level of these neurotransmitters was the main focus of clinical research. The biogenic amine hypothesis states that depression is caused by a deficiency of monoamines, particularly norepinephrine, dopamine and serotonin, and that it can be alleviated by the administration of antidepressant drugs that increase the availability of these neurotransmitters. As illustrated in Table 1, serotonin plays an important role in sleep, memory and anxiety. Norepinephrine, which is mainly related to attention and alertness, also continues to be a major focus in research. Another neurotransmitter of interest in depression is dopamine. It is well documented that this hormone is related to alertness, motivation, pleasure, and memory, as well as interest in life [16].

One way of increasing monoamines lies around the use of monoamine oxidase inhibitors (MAOIs) [17]. MAOIs are chemicals that inhibit the activity of the monoamine oxidase enzyme family. They are particularly effective in treating atypical depression [18]. Because of their ability to block the action of monoamine oxidase, which catalyzes the oxidative deamination of monoamines, they are able to significantly increase the availability of neurotransmitters in the brain. Other drugs, called tricyclic antidepressants (TCA), were developed in the 1950s in order to increase the level of neurotransmitters [19]. These endogenous chemicals transmit signals across a synapse from one neuron to another one. In the presynaptic neuron cell, there are several synaptic vesicles that contain neurotransmitters. They get released in the synaptic cleft and then get taken up by the postsynaptic cell of another neuron. TCA's mode of action involves blocking the process of reuptake by the postsynaptic cell, which increases the amount of neurotransmitter in the synaptic cleft.

There are several other molecules that play a role in depression, such as acetylcholine, histamine, and both opioid and non-opioid peptides. Understanding their anatomical and neurochemical interaction is crucial for the development of effective treatment of depression. The remaining of this article will discuss the effectiveness of antidepressants drugs and other molecules used to treat affective disorders, by illustrating their mode of action and their side effects.

\section{Monoamine Oxidase Inhibitors}

Monoamine oxidase inhibitors were the first type of antidepressant developed. They are a class of enzyme that selectively inhibits the activity of the monoamine oxidase enzyme family. Used in the early 1950s to treat tuberculosis, it was found that iproniazid had a significant mood-elevating effect, which encouraged scientists to

Table 1. Main neurotransmitters involved in depression and their physiological effects.

\begin{tabular}{cl}
\hline Neurotransmitter & \multicolumn{1}{c}{ Physiological Effects } \\
\hline Dopamine & Alertness, Attention, Clarity, Motivation, Working Memory \\
Norepinephrine & Attention, Concentration, Determination, Endurance \\
Serotonin & Satisfaction, Relaxation, Insomnia, Pleasure, Anxiety, Learning Memory \\
\hline
\end{tabular}


conduct clinical trials [20]. This enabled clinicians to discover its ability to inhibit monoamine oxidase, which led to the development of drugs having the same mode of action. MAOIs are currently used to treat the most challenging affective disorders, including those with atypical depression, bipolar depression and treatment-resistant depression [21], but are also useful for the treatment of panic attacks, posttraumatic stress and obsessive-compulsive disorder.

One example of a MOA is tranylcypromine (Parnate), which is very commonly prescribed in North America. Other MAOIs such as isocarboxazid (Marplan), phenelzine (Nardil) and Selegiline (Emsam) can also provide a safe treatment for depressed individuals [21]. The chemical classification of these drugs comprises the hydrazines and the non-hydrazine. Table 2 provides a list of the most commonly used MOAIs with their chemical classifications and most notable side effects.

These drugs are also divided into two groups, MAO-A inhibitors and MAO-B inhibitors, depending on their ability to inhibit the A or B form of the MAO enzyme. Both MAOs are crucial to the inactivation of monoaminergic neurotransmitters, and they both break down dopamine, tyramine, and tryptamine equally. However, these two isoenzymes display different specificities. While MAO-B breaks down phenethylamine and benzylamine, MAO-A is important for the catabolism of serotonin, melatonin, noradrenaline, adrenaline, and monoamines ingested in food. Like most antidepressants, MAOIs work by modifying the levels of one or more of the neurotransmitters naturally found in the brain. The MAOIs act on the monoamine oxidase enzyme in an irreversible manner, preventing their enzymatic deamination. Despite their relatively short plasma half-life, these drugs have a relatively long duration of action. Studies have shown that treatment with an MAOI increases the level of norepinephrine, serotonin and dopamine in rat brain for several weeks, which helps boost mood by improving brain cell communications.

A disadvantage of using MAOIs is that the patient needs to adhere to a strict diet since their use can cause a significant increase in blood pressure when taken with certain foods or other prescriptions. Patients treated with MOAIs also commonly report adverse signs such as hypertension, insomnia, frequent awakenings, reduced sleep time, weight gain and impaired sexual responses. Moreover, MAOIs can target non-specific enzymes, such as the cytochrome $\mathrm{P}_{450}$, which degrades a variety of drugs as well as biogenic amines. Significant drug interaction can also occur upon using their administration. Because of this, monoamine oxidase inhibitors are being replaced by other antidepressants, such as the tricyclic antidepressants, which are generally safer to use.

\section{Tricyclic and Second-Generation Antidepressants}

The tricyclic antidepressants (TCAs) were first discovered in the early 1950s and were subsequently introduced as antidepressant drugs [22]. Their name is derived from their chemical structure, which contains three rings of atoms. Although TCAs are chemical compounds that are mainly used as antidepressants, they are also effective in treating other medical disorders such as generalized anxiety and social phobia. Common tricyclic antidepressants include imipramine (Tofranil), amitriptyline (Elavil), amoxapine (Asendin) and protriptyline (Vivactyl). Several other newer antidepressants drugs (second-generation) have been developed in order to reduce the side effects and improve the efficacy. TCAs antidepressant effect is attributed to inhibition of the neuronal uptake mechanism that normally stops the action of neurotransmitters. The majority of the TCAs are serotonin-norepinephrine reuptake inhibitors. By inhibiting reuptake of neurotransmitters, TCAs help improve the communication between neuronal cells since the duration of neurotransmitter action at the synapse is prolonged [23]. Although being effective in treating depression, the use of TCA can cause several side effects. Some of the most frequent side effects experienced by patients are constipation, dry mouth, fatigue, tachycardia, weakness and urinary retention. For this reason, they have been increasingly replaced by newer antidepressants, which present fewer side effects. These new drugs are the second-generation antidepressants, which include the selective serotonin reuptake inhibitors, the serotonin-norepinephrine reuptake inhibitors, and the norepinephrine reuptake inhibitors. The term "Third generation antidepressant" is sometimes employed to refer to even newer antidepressants, which often include selective serotonin reuptake inhibitors such as fluoxetine (Prozac), paroxetine (Paxil) and sertraline (Zoloft) [24]. The second-generation drugs were introduced in the 1970s and they differ from the TCAs in their tricyclic nucleus that is largely modified. The earliest drugs in the market were iprindole (Prondol), doxepine (Sinequan) and amoxapine (Asendin). Moreover, two bicyclic compounds were developed in the mid-1970s with the ability to prevent the reuptake of serotonin without affecting other neurotransmitters. Examples include fluoxetine and citalopram. Table 3 provides a list of the most common tricyclic and second-generation antidepressants including their class and trade name [25]. 
Table 2. Most commonly prescribed monoamine oxidase inhibitors.

\begin{tabular}{lll}
\hline \multicolumn{1}{c}{ Chemical classifications } & \multicolumn{1}{c}{ Drugs } & \multicolumn{1}{c}{ Side Effects } \\
\hline Hydrazine & Isocarboxazid, Phenelzine, Selegiline & Headache, Vomiting, Nausea \\
Non-Hydrazines & Pargyline, Tranylcypromine & Blurred Vision, Dizziness, Vomiting \\
MAO-A Inhibitors & Clorgyline, Cimoxatone, Amiflamine & High Blood Pressure, Drug Interaction \\
MAO-B Inhibitors & Deprenyl, Caroxazone & Insomnia, Altered Heart Rythm \\
\hline
\end{tabular}

Table 3. Most common tricyclic and second-generation antidepressants.

\begin{tabular}{ll}
\hline \multicolumn{1}{c}{ Class } & \multicolumn{1}{c}{ Antidepressant Drugs } \\
\hline Classic Tricyclics & Amitriptyline (Elavil), Desipramine (Norpramine), Imipramine (Tofranil) \\
Modified Tricyclics & $\begin{array}{l}\text { Amoxapine (Asendin), Doxepine (Sinequan), Iprindole (Tertran), Trimipramine } \\
\text { (Surmontil), Protriptyline (Vivactyl) }\end{array}$ \\
Serotonin Reuptake Inhibitors & $\begin{array}{l}\text { Clomipramine (Anafranil), Citalopram (Cipramil), Fluoxetine (Prozac), Fluvoxamine } \\
\text { (Luvox), Sertraline (Zoloft), Venlafaxine (Effexor) }\end{array}$ \\
Atypical Antidepressants Trazodone (Serzone) & Bupropion (Wellbutrin), Maprotiline (Ludiomil), Mianserin (Tolvon),
\end{tabular}

Studies have shown that depressed patients treated with selective serotonin reuptake inhibitors, such as citalopram and clomipramine can anticipate a high probability of symptom improvement within few days [26]. Although the second-generation drugs can cause some side effects, they are more effective and safer that the TCAs and the MAOIs, especially when taken in large quantities.

\section{Mood Stabilizer: Clinical Action and Use in Bipolar Disorder}

Mood stabilizers are a group of medication used to treat mood disorders characterized by intense and sustained mood shifts. This class of medication is primarily used for treating patients who suffer from bipolar disorder, which is characterized by episode of mania or hypomania alternating with depression. During mania an individual feels abnormally happy, energetic, but most often have unrealistic ideas and makes poor decisions. Other symptoms include irritable mood, pressured speech, and decreased need for sleep [27]. Moreover, the suicide rate is higher than in patients affected with depression, and is estimated to be between $10 \%$ and $25 \%$. There are two main types of bipolar disorder: type I and type II. Type I is characterized by at least one manic episode, which can alternate with depression [28] [29]. In type II bipolar disorder, there are no manic episodes, but one or more hypomanic and major depressive episodes [28]. There are a number of pharmacological medications used to treat bipolar disorder [30], with Lithium being the most commonly. Not only does it treat acute manic episodes and prevent relapses, but is also effective in treating depression [31]. When it's administered to the patient, lithium becomes distributed in the central nervous system and interacts with several neurotransmitters such as serotonin. Animal studies have shown that when the serotonergic neurons from rat are treated with lithium, serotonin release is enhanced compared to no lithium treatment [32]. One of the disadvantage of using lithium is that is causes a variety of side effects. Because of its narrow therapeutic range (range between the therapeutically effective and toxic dose), drug monitoring is required to avoid toxicity. Signs and symptoms of toxicity include nausea, vomiting, diarrhea, and ataxia [33]. However, these symptoms can often be improved by lowering the dose of lithium [34].

A number of other pharmaceutical agents can also be used for treating bipolar disorders, which are useful especially for patients who do not respond well to lithium therapy or who cannot tolerate its side effects. Such drugs include the anticonvulsants, also known as antiepileptic drugs. Carbamazepine, which is typically used treating seizure disorders and neuropathic pain, can also be used as a second line treatment for bipolar disorder. However, it's a poor antidepressant that is less effective then lithium, and that often requires the simultaneous use of other antidepressants [35]. Carbamazepine was displaced by valproic acid (Depakote) in the 1990s. Since then, Depakote has become a commonly prescribed treatment, which is more suitable for treating patients with manic episodes [36]. Lamotrigine, another anticonvulsant, is also a good mood stabilizer that has been shown to effectively treat bipolar depression [37]. Other agents, such as the antipsychotic medications, may be effective for short-term treatment of bipolar manic episodes and appear to have a better efficacy than lithium and anti- 
convulsants [30]. Olanzapine and clozapine are both effective in treating bipolar disorder and preventing relapses. However, medications such as lithium are preferred when used for a long period of time [30].

\section{Conclusion}

Affective disorders are a group of disease with significant neurobiological consequences that involve functional and neuronal alterations in several regions of the brain. The biogenic amine hypothesis of depression has helped us understand the biological mechanisms and brain circuits involved in this disease, and have led to the development of several generations of antidepressants that include the monoamine oxidase inhibitors, the tricyclic and second-generation antidepressants, as well as other mood stabilizers like lithium and the anticonvulsants. Antidepressant drugs clearly have an effect not only on serotonin, dopamine and noradrenaline, but also on other molecules not discussed in this paper, such as histamine and acetylcholine. Such interactions are crucial in the understanding of the theory of depression and the effectiveness of antidepressants. Although antidepressant pharmacotherapy have shown to be very effective in the treatment of affective disorders, more work need to be done to optimize the therapeutic effects and reduce the side effects. Scientists need to emphasize on neuropharmacology research and on the development of novel treatments. Other potential treatments for depression are the use of electroconvulsive therapy, in which seizures are electrically induced, and deep brain stimulation, which sends electrical impulses to specific parts of the brain to provide relief from psychiatric illnesses. Finally, future research needs to focus on the identification of brain structures involved in mood disorders, and on the development of animal models, which will help identify the subtype of depression and optimize the effectiveness of pharmacological treatments.

\section{Financial Disclosure}

The author declares that they have no financial or other conflicts of interest to disclose.

\section{References}

[1] Weissman, M.M. and Boyd, J.D. (1984) The Epidemiology of Affective Disorders. Neurobiology of Mood Disorders. Williams and Wilkins, Baltimore, 60-75.

[2] Kenneson A, Funderburk JS, Maisto SA (2013) Substance Use Disorders Increase the Odds of Subsequent Mood Disorders. Drug and Alcohol Dependence, 133, 338-343. http://dx.doi.org/10.1016/j.drugalcdep.2013.06.011

[3] Marchand, R., Dilda, V. and Jensen, C.R. (2005) Neurobiology of Mood Disorders. Hosp Physician, 41, 17-26.

[4] American Psychiatric Association (2013) Diagnostic and Statistical Manual of Mental Disorders. 5th Edition, American Psychiatric Publishing, Arlington, 302.

[5] Brotman, A.W., Falk, W.E. and Gelemberg, A.J. (1987) Pharmacological Treatment of Acute Depressive Subtypes. In: Melter, H.Y., Ed., Psychopharmacology: The Third Generation of Progress, 1031-1040.

[6] Daniel, T.G., Daniel, L.S. and Daniel, M.W., eds. (2011) Psychology. 2nd Edition. Worth Publishers, New York, 564.

[7] Keller, M.B., Lavori, P.W., Endicott, J., Coryell, W. and Klerman, G.L. (1983) “Double Depression”: Two-Year Follow-Up. American Journal of Psychiatry, 140, 689-694.

[8] Chanaka, W. and Perminder, S. (2008) Treatment-Resistant Depression: Critique of Current Approaches. The Australian and New Zealand Journal of Psychiatry, 42, 751-762. http://dx.doi.org/10.1080/00048670802277206

[9] Kern, S., Rohleder, N., Eisenhofer, G., Lange, J. and Ziemssen, T. (2014) Time Matters-Acute Stress Response and Glucocorticoid Sensitivity in Early Multiple Sclerosis. Brain, Behavior, and Immunity.

[10] Kandel, E.R. (1991) Disorders of Mood: Depression, Mania, and Anxiety Disorders. In: Kandel, et al., Eds., Principles of Neural Science, Elsevier, New York, 869-886.

[11] Gold, P.W., Goodwin, F.K. and Chrousos, G.P. (1988) Clinical and Biochemical Manifestations of Depression. The New England Journal of Medicine, 319, 413-420. http://dx.doi.org/10.1056/NEJM198808183190706

[12] Hayes, P.E. and Ettigi, P. (1983) Dexamethasone Suppression Test in Diagnosis of Depressive Illness. Clinical Pharmacology, 2, 538-545.

[13] Reddy, M.S. and Kuruvilla, K. (1986) Dexamethasone Suppression Test in Depression. Indian Journal of Psychiatry, 28, 195-200.

[14] Brodie, B.B., Olin, J.S., Kuntzman, R.G. and Shore, P.A. (1957) Possible Interrelationship between Release of Brain Norepinephrine and Serotonin by Reserpine. Science, 125, 1293-1294. 
[15] Lee, M.J. and Wei, J.W. (2013) The Influences of Reserpine and Imipramine on the 5-HT2 Receptor Binding Site and Its Coupled Second Messenger in Rat Cerebral Cortex. The Chinese Journal of Physiology, 56, 199-208. http://dx.doi.org/10.1126/science.125.3261.1293

[16] Nutt, D.J. (2008) Relationship of Neurotransmitters to the Symptoms of Major Depressive Disorder. Journal of Clinical Psychiatry, 69, 4-7.

[17] Legoabe, L.J., Petzer, A. and Petzer, J.P. (2014) $\alpha$-Tetralone Derivatives as Inhibitors of Monoamine Oxidase. Bioorganic Medicinal Chemistry Letters, 24, 2758-2763. http://dx.doi.org/10.1016/j.bmcl.2014.04.021

[18] Singh, T. and Williams, K. (2006) Atypical Depression. Psychiatry (Edgmont), 3, 33-39.

[19] López-Muñoz, F. and Alamo, C. (2009) Monoaminergic Neurotransmission: The History of the Discovery of Antidepressants from 1950s until Today. Current Pharmaceutical Design, 15, 1563-1586. http://dx.doi.org/10.2174/138161209788168001

[20] Moncrieff, J. (2008) The Creation of the Concept of an Antidepressant: An Historical Analysis. Social Science Medicine, 66, 2346-2355. http://dx.doi.org/10.1016/j.socscimed.2008.01.047

[21] Shulman, K.I., Herrmann, N. and Walker, S.E. (2013) Current Place of Monoamine Oxidase Inhibitors in the Treatment of Depression. CNS Drugs, 27, 789-797. http://dx.doi.org/10.1007/s40263-013-0097-3

[22] Carson, V.B. (2000) Mental Health Nursing: The Nurse-Patient Journey. W.B. Saunders, Philadelphia, 423.

[23] Gillman, P.K. (2007) Tricyclic Antidepressant Pharmacology and Therapeutic Drug Interactions Updated. British Journal of Pharmacology, 151, 737-748. http://dx.doi.org/10.1038/sj.bjp.0707253

[24] Olver, J.S., Burrows, G.D. and Norman, T.R. (2001) Third-Generation Antidepressants: Do They Offer Advantages over the SSRIs? CNS Drugs, 15, 941-954. http://dx.doi.org/10.2165/00023210-200115120-00004

[25] Feldman, R.S., Meyer, J.S. and Quenzer, L.F. (1997) Principles of Neuropsychopharmacology. Sinauer Associates Inc., Sunderland, Massachusetts, 834.

[26] Dennehy, E.B., Marangell, L.B., Martinez, J., Balasubramani, G.K. and Wisniewski, S.R. (2014) Clinical and Functional Outcomes of Patients Who Experience Partial Response to Citalopram: Secondary Analysis of STAR*D. Journal of Psychiatric Practice, 20, 178-187. http://dx.doi.org/10.1097/01.pra.0000450317.76117.62

[27] American Psychiatric Association (1994) Diagnostic and Statistical Manual of Mental Disorder (DSM-IV). 4th Edition, APA, Washington DC.

[28] Barnett, J.H. and Smoller, J.W. (2009) The Genetics of Bipolar Disorder. Neuroscience, 164, 331-343. http://dx.doi.org/10.1016/j.neuroscience.2009.03.080

[29] Renk, K., White, R., Lauer, B.A., McSwiggan, M., Puff, J. and Lowell, A. (2014) Bipolar Disorder in Children. Psychiatry Journal, 2014, Article ID: 928685.

[30] Geddes, J.R. and Miklowitz, D.J. (2013) Treatment of Bipolar Disorder. The Lancet, 381, 1672-1682. http://dx.doi.org/10.1016/S0140-6736(13)60857-0

[31] Brown, K.M. and Tracy, D.K. (2013) Lithium: The Pharmacodynamic Actions of the Amazing Ion. Therapeutic Advances in Psychopharmacology, 3, 163-176. http://dx.doi.org/10.1177/2045125312471963

[32] Scheuch, K., Höltje, M., Budde, H., Lautenschlager, M., Heinz, A., Ahnert-Hilger, G. and Priller, J. (2010) Lithium Modulates Tryptophan Hydroxylase 2 Gene Expression and Serotonin Release in Primary Cultures of Serotonergic Raphe Neurons. Brain Research, 1307, 14-21. http://dx.doi.org/10.1016/j.brainres.2009.10.027

[33] Marmol, F. (2008) Lithium: Bipolar Disorder and Neurodegenerative Diseases Possible Cellular Mechanisms of the Therapeutic Effects of Lithium. Progress in Neuro-Psychopharmacology and Biological Psychiatry, 32, 1761-1771. http://dx.doi.org/10.1016/j.pnpbp.2008.08.012

[34] Kozier, B., Harvey, S. and Morgan-Samuel, H. (2008) Fundamentals of Nursing, Concepts, Process and Practice. Pearson Education, London, 189.

[35] Rapoport, S.I., Basselin, M., Kim, H.W. and Rao, J.S. (2009) Bipolar Disorder and Mechanisms of Action of Mood Stabilizers. Brain Research Reviews, 61, 185-209. http://dx.doi.org/10.1016/j.brainresrev.2009.06.003

[36] Bowden, C.L., Brugger, A.M., Swann, A.C., Calabrese, J.R., Janicak, P.G., Petty, F., Dilsaver, S.C., Davis, J.M., Rush, A.J. and Small, J.G. (1994) Efficacy of Divalproex Sodium vs Lithium and Placebo in the Treatment of Mania. Journal of the American Medical Association, 271, 918-924. http://dx.doi.org/10.1001/jama.1994.03510360044034

[37] Katayama, Y., Terao, T., Kamei, K., Hatano, K., Kohno, K., Makino, M., Mizokami, Y., Kodama, K. and Itoh, H. (2014) Therapeutic Window of Lamotrigine for Mood Disorders: A Naturalistic Retrospective Study. Pharmacopsychiatry, 47, 111-114. http://dx.doi.org/10.1055/s-0034-1375618 